\title{
The Intersection of Identity and Performing Arts for Black Physicists
}

\author{
Tamia Williams, ${ }^{1}$ Simone Hyater-Adams, ${ }^{2}$ Kathleen Hinko, ${ }^{3}$ Claudia Fracchiolla ${ }^{4}$ Kerstin Nordstrom, ${ }^{1}$ and Noah Finkelstein ${ }^{5}$ \\ ${ }^{1}$ Department of Physics, Mount Holyoke College, South Hadley, MA, USA \\ ${ }^{2}$ ATLAS Institute, University of Colorado Boulder, Boulder, CO, USA \\ ${ }^{3}$ Department of Physics, Lyman Briggs College, Michigan State University, East Lansing, MI, USA \\ ${ }^{4}$ School of Education, University College Dublin, Dublin, Ireland \\ ${ }^{5}$ Department of Physics, University of Colorado Boulder, Boulder, CO, USA
}

\begin{abstract}
In an effort to counter the systemic marginalization of Black students in physics, we consider the role performing arts plays in the positive development of science identity. In this work, building on previous studies of physics and racial identities, and studies that demonstrate the positive role of arts for underrepresented groups in STEM, we collect and analyze interviews of thirteen Black physicists. We identify themes that relate to the ways in which Black physicists participate in the performing arts, and map those themes onto the previouslydeveloped Critical Physics Identity (CPI) framework. We find that the performing arts can have positive impacts for Black physicistsâ identities by serving as a material resource, and can activate ideational resources, which support relational resources or enhance a personâs sense of performance/ competence.
\end{abstract}

\section{INTRODUCTION}

In the United States, Black people are underrepresented in the field of physics [1]. There has been research that shows the disparities of underrepresentation caused or exacerbated by aspects of the culture of physics that marginalizes Black people $[2,3]$. They can be affected by multiple forms of racial bias such as implicit bias, racial microaggressions, or racial stereotypes, such as assumptions and myths about the academic abilities of certain groups of students, especially Black students, or feelings of being personally diminished by White teachers or peers [3]. Navigating this culture may sway individuals into thinking that they do not fit the criteria of a "typical" physicist. We know that how one negotiates their physics identity is crucial to becoming a physicist and maintaining membership in the physics community [4]. Therefore, to improve the culture of the physics community and increase the presence of minorities, especially Black individuals, there must be a further examination of physics identity.

Despite roadblocks the physics field presents, including privileged norms that favor competitive norms, traditionally associated with White male physicists, there have been studies that show underrepresented individuals made an effort to pursue STEM fields [4,5]. Counterspaces, a nontraditional activity that lies outside mainstream educational spaces, offer support and enhance feelings of belonging in STEM [5] making it possible for individuals to pursue STEM careers. For example conferences and campus-based groups that focus on STEM diversity can be considered counterspaces [5]. Creating counterspaces promotes learning wherein students' experiences are validated and viewed as critical knowledge [5]. Performing arts can be considered a counterspace. Understanding that performing arts can be a counterspace is very important in the rationale for the inclusion of arts in STEM education (STEAM). Some studies show that the arts can act as an identity mediator or coping mechanism for underrepresented groups in STEM [6,7]. However, there is a lack of research in understanding the intersection of physics, the arts, and identity, especially for those who identify as being Black.
In this study we seek to understand how performing arts supports Black physicists by: 1) Determining how and why Black physicists participate in the performing arts, and 2) characterizing the impact of performing arts on their physics identities. To achieve these research objectives, we apply the critical physics identity framework, along with emergent coding for performing arts participation, to interviews of Black physicists and report on our findings.

\section{BACKGROUND AND FRAMEWORK}

We use the Critical Physics Identity (CPI) framework [8], which expands on the constructs from both, Hazari et al.'s physics identity framework (recognition, interest, performance/competence) [9] and Nasir's racialized identities framework (relational, material, ideational) [10]. The CPI framework illuminates how physics identity differs for physicists across all racial and ethnic backgrounds and presents a more comprehensive understanding of the facets of identity that are relevant in a physics identity. Using the CPI framework helps us understand the ways in which the performing arts impact Black physicists and also broadens our concept of physics identity to include impact from the performing arts.

There are six constructs that make up the CPI framework [8]: relational resources, an aspect of a relationship with others in the context can impact one's connection to physics; material resources, material things that can provide access to other identity constructs; ideational resources, aspects of an idea that impact one's connection to physics; recognition, being recognized (or not) as a physicist or physics person; interest, interest in the physics field; performance/competence, one's beliefs in their ability to understand physics content and perform required physics tasks. Each of these constructs have both positive and negative subcodes, with the exception of our ideational resource which has internal and external subcodes.

When we discuss the role of arts in this paper, we mean that of the performing arts, music, dance and theatre arts. One particular reason why we focus on performing arts rather than vi- 
sual arts is because in visual arts, there is a tangible media that can show a person's emotions or feelings, in comparison to performing arts where the individual must express emotions and feelings through physical actions. The arts make ideas and theories, particularly complex ones, seem more comprehensible to students as more active involvement in learning is engendered [11]. Due to these reasons, we focus solely on the performing arts. In this work we examine the intersection of physics and art identity, the positive impacts of performing arts, and the overlap among constructs of identities.

\section{METHODS, DATA \& ANALYSIS}

In order to investigate how the performing arts can support physics identities, we collected interviews with Black physicists from a variety of backgrounds. Individuals in our data set identified as Black, African American, Caribbean and/or African. These individuals were sought out at national conferences, recruited via Black physicists organizations and/or worked with us at our various institutions. It is to be noted that even though participants may be from different nationalities all research is being done in the US and within US context. For this study, we defined a "physicist" as an individual who is close to obtaining or has already obtained a bachelor's degree in physics. In total, we collected thirteen interviews.

We developed an interview protocol that prompted interviewees to tell their experiences and what it means to be a physicist. The interviews were semi-structured, which gives the interviewees the liberty to focus on their physics and artistic identity intersections without the interview being too rigid. The second author, a Black woman physicist conducted all the interviews used for this research, and it is likely that this impacted the types of answers participants felt comfortable sharing. Some questions included in the interview protocol were: "Have you ever participated in any performance art in your past or currently?", "In what ways has participating in the arts benefited you?" and "What have you gained from those experiences and has it played a role in becoming a physicist?" After the interviews were conducted the participants were asked to complete a demographic survey, so that the questions regarding demographics in the interview did not impact the participants overall interview. Audio files of the interviews were transcribed by a third party. The second author validated all interviews after they were transcribed so that they were valid for our analysis process.

Interviews were approached with a narrative inquiry lens [12], which privileges the experience of the subject. Our analysis process started with two individuals coding the interviews with the CPI framework constructs and STEAM codes. All interviews were coded by two raters, once the coding was finished consensus was always reached through discussion if there were any coding discrepancies. Our coding team consisted of a White woman, a Latina woman and two African American women; this diverse team allowed us to discuss and minimize bias in our coding results.
We emphasize here that we use the CPI framework as it was originally developed: using operationalized definitions of the CPI constructs, we coded segments of the narrative where the subject talks about their journey in physics. The STEAM code that we used in this study is not a part of the CPI framework. Rather it is a separate code to classify sections of our interviews where we ask questions about the performing arts. The point of coding certain sections of the interview as STEAM was to look at the co-occurrences (using MAXQDA software) with the CPI codes that show up in the sections of the interviews that talk about the arts. Any CPI codes that are present in the STEAM coded segments are evidence that the subject was talking about their physics journey while also discussing the arts. Looking at the intersection of the STEAM codes and the CPI framework gave us a way of thinking about how these physicists engage with performing arts as part of their physics identity.

\section{RESULTS}

\section{A. Intersection of Performing Arts and Physics Identity}

Figure 1 shows the total number of counts where the CPI framework codes overlapped with the STEAM code. Below we provide examples of these overlapping results and discuss their meaning. It is to be noted that because we interviewed 13 individuals having 7 counts of a CPI code [i.e. relational resource] it is a significant amount of data.

Individuals who had the relational resource CPI code overlapping with the STEAM code, in their interviews, indicated that they meet different people by participating in the arts and found a community within their various performing arts disciplines.

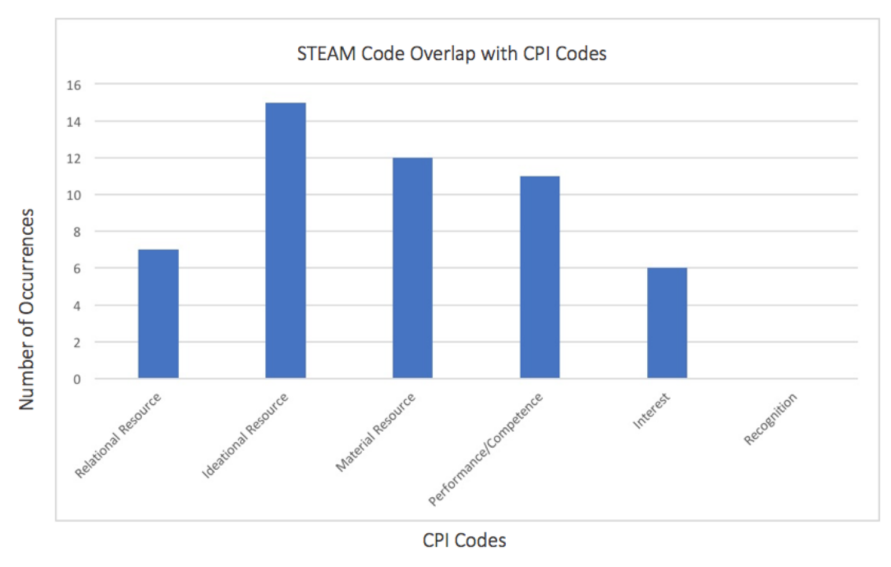

FIG. 1. Graph of the frequency of our CPI codes that overlapped with the STEAM code.

For example, Taylor states:

"I think being involved in arts and I guess the spoken word things that I do sometimes, that just helps me relate to people." 
From this example, we see the performing arts can produce meaningful relationships, which may be unlikely to find within physics. These relationships provide a different sense of identity that is not physics or occupational-related.

The overlapping of the ideational resource and STEAM codes means that individuals are talking about what's valued in physics, personal characteristics, positioning in physics and perception of physicists when discussing the performing arts. An example is when Riley compares physics with the performing arts:

"..for each [discipline] you need a certain sense
of an open mindedness and creativity to both,
and imagination to visualize things, and kind of
a will to explore. So I think each of them has
kind of offered that same opportunity to create,
in a way, or to learn, to explore the curiosity
that I have about the world, and yourself re-
ally, because you're finding a connection with
the world."

From this example, we infer that the performing arts can provide opportunities to create, visualize and explore the connections to the world similar to those that offered by the study of physics. Additionally, the activities of creation and exploration are in fact valued in both the performing arts and physics.

Individuals had a wide range of discussions of how the performing arts served as a material resource. For example Charlie states:

"..the way that you have to think about music is also the way that you have to think about doing science. I'm not, I don't know whether it's the art that supports you in becoming a physicist or whether it's a different manifestation of the way that your mind works."

Charlie found that the way they thought about music was also very similar to the way they thought about physics. This example also highlights the unique intersection of the content of physics and the performing arts.

Finally, we found that there were no recognition codes that overlapped with our STEAM code across any of our interviews. This result was one of the most surprising to come out of our analysis. Based on our previous work [13], recognition is a major factor in physics identity and is strongly connected to the resource constructs. Our definition of recognition is "being recognized as a physicist or physics person." We concluded that for the physicists in our data set tit was true that they were not recognized as physicists within these performing arts fields.

\section{B. Positive Influence of the Performing Arts}

We were able to indicate whether the CPI codes found to overlap with the STEAM codes were positive or negative influences on the individuals' pathway into physics. We found that overall, for individuals involved in performing arts, their participation in the arts positively impacted their pathway into physics. The relational resource code had 7 counts though out our data set with 3 being positive. The other 4 counts were coded as neither, showing some individuals had general interacts with people in the arts. Individuals who had formed positive relationships through the arts could indirectly strengthen their physics identity. After being prompted with the question of "Could you see yourself in the field of physics without having participated in the arts?" Avery expressed that participating in the arts opened them up to meeting new people; this response indicated that the arts helped this individual to be confident when it came to forming relationships in the physics community. The performance/competence code had 11 counts though out our data set with 6 being positive and showing a significant connection back to physics. The other 5 counts were coded as neither showing no connection back to their artistic identity. Riley states that they were able to think about physics in a creative way. They go on to say that knowing how inquisitive a person can be within the arts also allows a person to explore and ask questions in physics. For material resources code had 12 counts though out our data set with 5 counts being positive and 6 counts coded as neither and 1 count that was negative. Anytime a person talked about specific performance arts experiences and the impact that it had on their life, it was coded as positive. What we gather is that the performing arts act as a material resource and impact their personal characteristics about these Black physicist and also create relationships in the arts and in physics.

The individual with the negative material resource count, believed that this resource had absolutely no influence on their physics identity. However, this belief does not rule out the idea of the arts being a counterspace. They state how they like to keep music and physics separate, because it's "cheesy" and this leads people analyzing music too much. They would rather love music for it just being music and vice versa with physics.

\section{Connections between CPI Codes}

During our analysis process we saw the codes beginning to corelate with one another during the interviewees' narratives. One code connection was how a material resource provided skills to support a person's performance/competence. For example, in Figure 2, Jordan states that they grew up participating in the performing arts, such as church plays. What they gained from participating in these performing arts activities was a sense of confidence and oratory skills. They go on to iterate that physics never taught them how to project and work with an audience. These mentions of confidence and skills are all personal characteristics that this individual found from doing the arts. Lastly, the interviewee found that the two fields intertwine with one another and that the skills they learned in physics were used from the arts and that the skills learned in the arts were used in physics. This code con- 
nection tells us that people use the performing arts as material resources which leads them to personal ideational resources and support their performance/competence.

\begin{tabular}{|c|c|}
\hline $\begin{array}{l}\text { Material } \\
\text { Resource }\end{array}$ & $\begin{array}{l}\text { Yeah. If you grew up in a black church then you were in the church play. } \\
\text { The Christmas play and the Easter play, you were in those and you were } \\
\text { not allowed to leave. You had to be in it. So yes, I've been a part of like } \\
\text { those. }\end{array}$ \\
\hline $\begin{array}{l}\text { Ideational } \\
\text { Resource } \\
\text { (Personal Characteristic) }\end{array}$ & $\begin{array}{l}\text { Sense of confidence, I will say that. Sense of confidence. I mean like oratory } \\
\text { skills and things of that- oratory skills. Because if you're going to do poetry a } \\
\text { lot of those things require you to get up on the stage and you're having to } \\
\text { present yourself, you know, in front of a huge audience, and I have learned so } \\
\text { much about, you know, how to project and work with the audience } \\
\text { because of these, you know, because of the plays and things of that nature. } \\
\text { I've learned a lot because of that. Physics never taught me how to project } \\
\text { and do that as a teacher and things like that. }\end{array}$ \\
\hline $\begin{array}{l}\text { Competence/ } \\
\text { Performance }\end{array}$ & $\begin{array}{l}\text { Oh man, I never thought of a correlation between the two. I don't know. I don't } \\
\text { know. I don't want to say they're two separate worlds, they're not two } \\
\text { separate worlds, but it's just kind of like they just interweave each other. I } \\
\text { feel like I use skills that I've learned from the theater in my physics and I } \\
\text { use, you know, my thought and analytical skills within my, you know, if } \\
\text { I'm doing something for a play or something like that. I don't know. I think } \\
\text { I use both interchangeably. }\end{array}$ \\
\hline
\end{tabular}

FIG. 2. A coded example from Jordan's interview of the code connection from Material resource, to ideational resource, to competence/performance.

\begin{tabular}{|c|c|}
\hline $\begin{array}{l}\text { Material } \\
\text { Resource }\end{array}$ & $\begin{array}{l}\text { But I would really say that sort of doing performance art. The sort of more substantive } \\
\text { thing that I've done recently is the plat that I did as part of a [performance group from } \\
\text { Harlem]. }\end{array}$ \\
\hline $\begin{array}{l}\text { Ideational } \\
\text { Resource } \\
\text { (Personal Characteristic) }\end{array}$ & $\begin{array}{l}\text { And so I was thinking about doing something different in the winter term once I found out } \\
\text { that I was being recommended for tenure, and this opportunity presented itself and I } \\
\text { couldn't think of a reason not to do it other than that that I was afraid, and so I decided, I took } \\
\text { my mom's advice and said that's not a reason to not do it and so I'm going to challenge myself } \\
\text { and do this despite my fear. And so this served as an opportunity to challenge myself in a } \\
\text { different wat than I've challenged myself before, and I was successful. And so that was } \\
\text { encouraging. }\end{array}$ \\
\hline $\begin{array}{l}\text { Relational } \\
\text { Resource }\end{array}$ & $\begin{array}{l}\text { And I feel more connected to the students, at least those students, because we now have that } \\
\text { common experience together. And they were being--So the physics department is on the second } \\
\text { and third floor of one of the buildings and there's no reason to come up here unless you're taking } \\
\text { physics, and there aren't a lot of minority student who take the physics courses, and so it was a } \\
\text { great opportunity for me to meet some of the black students on campus and also see what the } \\
\text { issues were that I kind of peripherally heard about bit wasn't really sure what was going on. }\end{array}$ \\
\hline
\end{tabular}

FIG. 3. A coded example from Charlie's interview of the code connection from material resource, to ideational resource, to relational resource.

The other code connection that we found in our analysis was how material resources are avenues for relational resources to form (Figure 3). We find that material resources again lead to internal ideational personal characteristics, which then impacts the way they form relationships with other people. These relationships are often very positive when it comes to connecting with other people in physics. For example, Charlie states how they recently participated in a play. This opportunity came at a time when they were being recommended for tenure and also at a time where they could not turn this opportunity down. They knew that participating in the play would challenge them and help them face their fears. A byproduct of their participation was deeper connections to their students.

\section{DISCUSSION AND FUTURE WORK}

In this work, we found that the performing arts can have positive impacts for Black physicists' physics identity. We see how the performing arts can be used as a material resource, much like science programs or outreach. As a material resource, the performing arts can activate ideational resources, which translates into avenues for relational resources or enhance a person's performance/competence.

The use of the CPI framework and classifying the performing arts section of our interview as STEAM helped to tell a different narrative of how the arts are supporting Black physicists rather than the arts being treated as a tool to teach physics content. We hope that in analyzing these interviews it serves as guidelines in designing informal programs in the future that incorporate arts, not for the content knowledge but the self expression aspect of it. Furthermore, seeing how the arts have supported Black physicists we hope that this work can be used as a stepping stone to learn if and how the arts can support physics identity for other marginalized groups.

In future work, we will continue finding Black physicists who want to share their stories on their involvement in the arts. These stories will help broaden our results and either solidify or challenge the results that we have presented here.

\section{ACKNOWLEDGMENTS}

We thank Mount Holyoke College for supporting travel. This work was also supported by NSF Award \#1423496 \& NSF \#DMR 1548924.
[1] Czujko, R., Ivie, R., \& Stith, J. H. (2008). AIP report Number R-444.

[2] Grap.APS Edu. \& Dive., Phys.Deg.3-yr aver. (20132015),IPEDS.Compl.Sur.

[3] McGee, E. O. Amer.Edu.Res.J., 53(6) (2016).

[4] Wenger, E. Social learning systems and communities of practice(Springer, London,2010). pp. 179-198.

[5] Ong, M.,et al. J. Res. Sci. Teach., 5522 (2017).

[6] James, Jon. 'The PERF. Proj: Using Perform. Arts Incre. Engage Under. Sci.” FEMS Micr. Let., vol. 364, no. 8, Jan. 2017.
[7] Mejia D., Ms Thesis, Smith College, 2012.

[8] Hyater-Adams, S., et al. Phys.Rev.Phys.Edu.Res., 141 (2018).

[9] Nasir, N. Racialized identities: Race and achievement among African American youth. (Stanford University Press, 2012).

[10] Hazari, Z., et al. J. Res. Sci. Teach., 478 (2010).

[11] Williams, T. et al. Poster: in AAPT/PERC Conference, 2017.

[12] Connelly, F. M., et al. Edu. Res., 195 (1990).

[13] Hyater-Adams, S.,et al. PERC proceedings, 2016. 